\title{
Monolithically suspended fused silica substrates with very high mechanical $Q$
}

\author{
S. Traeger ${ }^{1}$, B. Willke ${ }^{2}$, K. Danzmann ${ }^{3}$ \\ Institut für Atom- und Molekülphysik, Universität Hannover, Appelstrasse 2, D-30167 Hannover, Germany
}

Received 22 July 1996; revised manuscript received 11 October 1996; accepted for publication 1 November 1996

Communicated by P.R. Holland

\begin{abstract}
To minimize thermal noise in gravitational-wave detectors high mechanical quality factors are required for the suspended test masses. Monolithic suspensions are proposed for advanced detectors because of their low losses in all mechanical modes. We investigated the influence of preparing these suspensions on internal $Q$ 's of suspended substrates.
\end{abstract}

PACS: 04.80; 95.55

Keywords: Gravitational wave detector; Thermal noise; Monolithic suspension

\section{Introduction}

Long baseline Michelson-type interferometers for the direct observation of gravitational waves are currently under construction [1-3]. The sensitivity of these detectors will be limited by photon shot noise at frequencies above $1 \mathrm{kHz}$ and by seismic noise at frequencies below $10 \mathrm{~Hz}$. Off-resonant thermal noise will be the limiting noise source in between. According to the fluctuation-dissipation theorem, the quality factors of the mechanical modes of the test masses should be as high as possible to lower the thermal noise power spectral density in this frequency domain [4].

To reduce seismic disturbances the test masses of a laser-interferometric gravitational-wave detector will be suspended as pendulums. These suspensions show thermal noise in the frequency band of interest as well

\footnotetext{
${ }^{1}$ E-mail: Traeger@mpq.mpg.de.

2 E-mail: Willke@mpq.mpg.de.

${ }^{3}$ E-mail: Danzmann @mpq.mpg.de.
}

as do the test masses themselves. High mechanical quality factors are required for the substrates of the test masses as well as for their suspensions. If the substrates are suspended on wire slings, dissipation of mechanical energy occurs due to friction at the breakaway points of the wires at the substrates and at the pendulum support structure. This dissipation leads to lowering of the mechanical $Q$ 's although high quality factors for the internal modes of the test masses suspended on steel wire slings have been obtained $[6,7]$.

By far the highest quality factors have been reported for the pendulum mode of a monolithic fused silica suspension by Braginsky et al. [5], but there has always been the suspicion that this type of suspension might adversely affect the $Q$-values of the internal modes of the test mass. This question is the subject of our investigation. We suspended high quality synthetic fused silica substrates on fused silica fibres with emphasis on a very high mechanical quality factor for the internal modes of the substrates. $Q$ values of 
$5.2 \times 10^{6}$ have been measured. In this paper preparation methods for these suspensions and an experiment to measure their internal mechanical quality factor are presented. The influence of bonding techniques on the internal mechanical quality factors of the suspended substrates is discussed.

\section{Experimental arrangement}

We determined the mechanical quality factor of the monolithically suspended fused silica substrates interferometrically using a suspended substrate as one end mirror of a Michelson-type interferometer. The second end mirror was mounted on a piezoelectrical transducer. A servo loop locked the output of the interferometer to the half-intensity point by moving the second end mirror. The corner frequency of this servo loop was at $60 \mathrm{~Hz}$. The resonance frequency of the bending mode which was the lowest mode of the suspended substrate $(25.4 \mathrm{~mm}$ in diameter, $12.1 \mathrm{~mm}$ in thickness) was of the order of $100 \mathrm{kHz}$. Thus, intensity modulation of the signal at the output port of the interferometer generated by vibration of the substrate at its resonance frequency can be detected by a photodiode and measured with a lock-in amplifier.

We excited the substrate's internal vibrational mode at resonance with an electrostatic drive [6]. The electrode of this drive was connected to a signal generator via a high-voltage amplifier. The generator was locked to a rubidium oscillator with an Allan variance of $\sigma(1 s)=10^{-11}$. It provided the source for the field we used to excite the substrate's vibration as well as the local oscillator for the lock-in amplifier we used to measure the generated signal. The lock-in amplifier was used in the quadrature mode giving magnitude and phase of the signal. It was, unfortunately, not possible to measure higher order modes of the substrates we used with this sensing system. The next higher mode, which in this case is the fundamental longitudinal mode, would occur around $140 \mathrm{kHz}$ which is out of the range of the lock-in amplifier. It is known that a widely varying of the $Q$ can be found for various modes. This is probably due to different coupling between the vibration of the substrate and the suspension wire for different internal modes. In this respect the mode under investigation here should be one of the worst because the fibre is attached to the substrate at a point where the displacement of the first order bending mode cannot vanish.

After switching off the connection between signal generator and high-voltage amplifier the decay time of the free oscillation was measured. This measurement was repeated several times and the decay times were averaged. The mechanical quality factor was calculated from the relation

$Q=\pi \times \tau \times f_{0}$,

where $\tau$ is the time the amplitude of the vibration needs to fall to the $\mathrm{e}^{-1}$ th part and $f_{0}$ is the resonance frequency of the mode.

Excited by seismic noise, the amplitudes of motion of the suspension pendulum at its resonance frequencies were higher then the dynamic range of the servo loop locking the interferometer. Hence, additional local control servo loops were required to damp the pendulum motion. For these servo loops we used electrostatic actuators. By using these actuators the problems of attaching magnets to the substrates [7] are avoided. To achieve sufficient forces with the actuators very inhomogeneous electrostatic fields as well as high voltage were needed. We used sharp electrodes which were connected to $300 \mathrm{~V}$ high-voltage amplifiers. Since electrostatic actuators can only pull, we used sets of electrodes on opposite sides of the substrate.

It is important to note that our arrangement of the local control servo loops allowed us to made the ringdown measurements when the loops were closed. Once the pendulum was damped the fields applied to the substrate nearly vanished and hence the capacitive damping was negligible. This is due to the fact that with our arrangement no bias was needed. However, a bias of $150 \mathrm{~V}$ was applied onto the additional electrode we used to excite the internal mode of the substrate. The calculated capacitive damping due to this field was orders of magnitude less then the measured internal damping and thus capacitive damping was not a limiting dissipation process in the experiment. A schematic diagram of the experimental setup can be seen in Fig. 1.

The measurements were made in vacuum of $10^{-6}$ mbar. To ensure that seismic noise and vibrations of the vacuum pumps did not excite high frequency violin modes [8], we integrated seismic isolation stacks in 


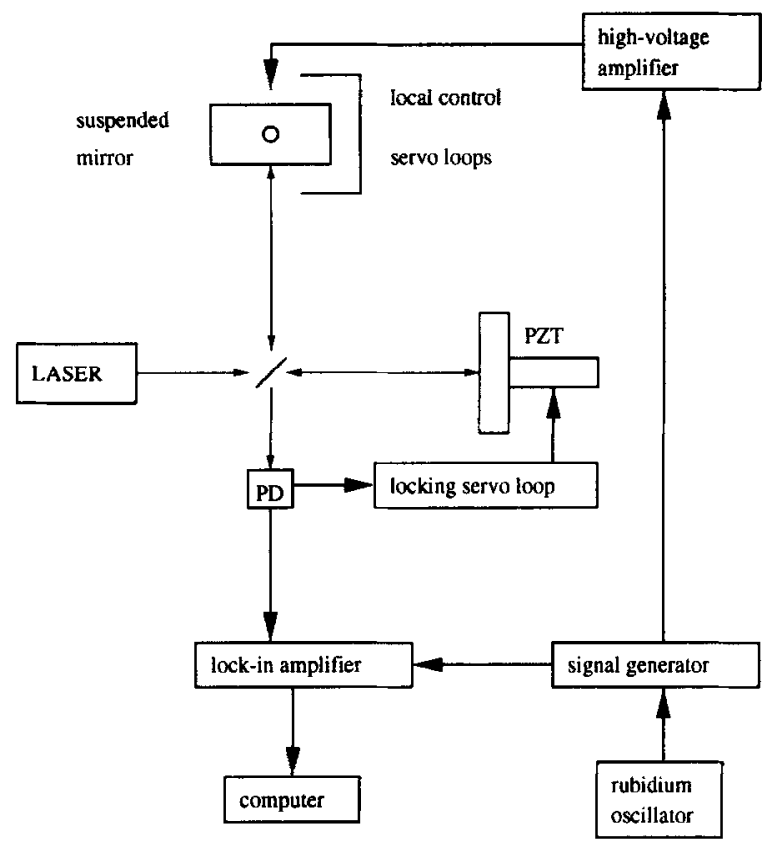

Fig. 1. Schematic diagram of the experimental arrangement used to measure the internal mechanical quality factor of substrates monolithically suspended on single fibres.

the four legs of the pendulum support structure. Every stack consisted of three layers of RTV-rubbers separated by lead bricks. A fused silica slab was clamped to the top plate of the support structure and the upper end of the pendulum wire was welded to this slab. We measured the transfer function of the isolation system. The normal modes occur at $10 \mathrm{~Hz}, 36 \mathrm{~Hz}$ and $73 \mathrm{~Hz}$. At the higher frequency regime this isolation system leeds to reduction of seismic noise at the suspension point.

\section{Bonding techniques}

We used fused silica optical fibre with its cladding stripped as the suspension wire. The diameter of the fibre was $120 \mu \mathrm{m}$. As reported by Logan et al. [9] the measured quality factor of a suspended test mass can be limited by coupling between internal modes of the substrate and resonances in the suspension wire. The relevant internal mode under investigation in our case was the first order bending mode. If the substrate vibrates in this mode then there is a horizontal displacement of the attachment point of the fibre to the substrate. That leads to bending of the fibre rather then to excitation of the fibre's longitudinal vertical modes as it is observed for a substrate vibrating in the fundamental longitudinal mode. If the resonances of the substrate and the fibre are at the same frequency, the energy of the substrate's internal motion disappears into the low $Q$ pendulum support structure.

Because of their high $Q$ the bandwidths of these resonances are very small. The resonance frequencies of the fibre depend on its length, so by adjusting the length of the fibre it is possible to mismatch the resonances of fibre and substrate.

The substrates were made of Suprasil 1, a highpurity synthetic fused silica from Heraeus. They were optically polished at their front ends as well as on their circumferences. Before preparing the suspensions, the substrates were cleaned with pure acetone to remove pollution from the surfaces. The substrates were suspended by single fibres which were connected to the circumferences. The connection was realized using a small hydrogen-burner. The temperature of the flame was $3200^{\circ} \mathrm{C}$.

We investigated three methods of bonding fibres to the substrates:

- Method 1: fusing the tibre to the circumference directly.

- Method 2: producing a pin on the substrate circumference by preshaping the substrate with diamond tools and welding the fibre to the pin.

- Method 3: producing a pin on the substrate circumference with the burner and welding the fibre to the pin.

In method 1 we heated the substrate circumference with the burner and contacted the fibre to the substrate when the circumference was melted.

In method 2 we preshaped the substrate circumference to get a small pin with length and diameter of $1 \mathrm{~mm}$. We used diamond tools with grain size of 15 $\mu \mathrm{m}$ to preshape the circumference. The substrate and the diamond tools were cooled with water. The substrate was cleaned after this treatment and the fibre was welded to the pin. Using this method only the small pin needed to be melted to connect the fibre to the substrate. The thermal stress in the whole bulk was lower then by using method 1 . This expectation was supported by qualitative observation of stress induced birefringence of the substrate between crossed polarizers. 
In method 3 the preshaping was not necessary. We prepared the fused silica pin first by pulling a small piece from a fused silica rod. The length of the piece was $1 \mathrm{~mm}$ and the diameter was around $300 \mu \mathrm{m}$. The pin was placed at the circumference and then only the small pin was heated with the burner. When the pin was hot, the top layers of the circumference started to melt and both fused together. It is important to note that with this method no direct interaction between the flame of the burner and the substrate itself took place. The heating of the bonding zone between the substrate and the additional pin was pretty smooth. We expect that the maximum temperature in this area was only slightly above the transformation temperature of Suprasil 1 which is at $1600^{\circ} \mathrm{C}$. The remainder of the rod was removed using the burner and the fibre was fused to the pin.

The advantage of having the pin on the substrate and of fusing the fibre to this pin is to have a welldefined contacting point. The thermal stress in the bulk was lower in method 2 than in method 1 and lowest in method 3. In method 3 additional mechanical treatment to the substrate as well as direct interaction between the flame of the burner and the substrate itself was avoided.

The aim of our work was to investigate bonding methods that allowed one to monolithically suspend substrates made from high quality synthetic fused silica on wires made from the same material having very low internal losses. However, to compare the monolithic suspensions with a nonmonolithic synthetic fused silica suspension we glued a fibre to the substrate. We used epoxy as adhesive and we did not heat the adhesive or treat it additionally. Unfortunately, the successful techniques to suspend substrates in steel wire slings [6-8] cannot be applied to suspend substrates on synthetic fused silica wires. Thus, we decided to glue the fibre onto the substrate even if almost certainly the epoxy itself was the dominant source of dissipation.

We tested method 2 on a much larger fused silica substrate of $64 \mathrm{~mm}$ in diameter and $70 \mathrm{~mm}$ long. We were able to weld fused silica rods onto the substrate without cracking of the substrate even if it was not annealed after the welding process. This seems to be promising for the possibility of fusing fibres to the large test masses of full-scale laser-interferometric gravitational-wave detectors. In the GEO600 team the methods described above as well as a mixture of it with other techniques like optical contacting will be tested on large-scale test masses within the next future $\lceil 10\rceil$.

\section{Results}

After bonding a fibre to it, the substrate was suspended as a pendulum. The internal vibrational mode of the substrate was excited and the decay trace was measured. This was repeated several times. The mechanical quality factor was calculated using the averaged decay time.

With method 1 the area of the substrate that needs to be melted is very large and the thermal stress in the bulk is high. We measured the lowest mechanical quality factors for the monolithically suspended substrates if the fibres were connected to the substrates by using this method. The $Q$ varied from $5.9 \times 10^{5}$ to $6.6 \times 10^{5}$.

With method 2 it was possible to control the conditions of the bonding process very well. The area that needs to be melted is well defined. The thermal stress is low because heating of the bulk itself is not necessary. To test the reproduceability of this method we connected three fibres to the same substrate. We fused the first fibre to the pin and measured the internal $Q$ of the substrate. The $Q$ was $1.0 \times 10^{6}$. Then we removed the fibre and replaced it with a new one. The $Q$ of the substrate was now $1.4 \times 10^{6}$. We replaced this fibre by a third one and measured the internal $Q$ to be $1.3 \times 10^{6}$.

To investigate the influence of treating substrates with diamond tools we cut the whole polished circumference with coarse tools of $30 \mu \mathrm{m}$ grain size. An internal $Q$ of $9.4 \times 10^{5}$ was measured. Then we replaced this fibre by a new one and measured the internal $Q$ to be $1.1 \times 10^{6}$. A significant lowering of the internal $Q$ after destroying the polished surface was not found. This shows that the internal mechanical $Q$ 's of our monolithically suspended substrates were not limited by surface losses.

We obtained the highest internal $Q$ if we followed method 3 in the bonding process. The averaged $Q$ was $5.2 \times 10^{6}$. We believe that the $Q$ was limited by losses into bending of the bonding zone and of the fibre although the resonance frequencies of the 
Table 1

Experimental data of the shown decay traces

\begin{tabular}{lllll}
\hline Trace & Method & $f_{0}[\mathrm{~Hz}]$ & $\tau[\mathrm{s}]$ & $Q_{\text {intern }}$ \\
\hline A & glued & 100296 & 0.8 & $2.5 \times 10^{5}$ \\
B & 1 & 100381 & 2.1 & $6.6 \times 10^{5}$ \\
C & 2 & 101055 & 4.5 & $1.4 \times 10^{6}$ \\
D & 3 & 100819 & 16.4 & $5.2 \times 10^{6}$ \\
\hline
\end{tabular}

substrate and the fibre were mismatched as described above. Because the bonding pin and the end of the fibre were melted when they were fused together there was thermal stress in this small area. That can lead to lowering of the intrinsic material quality factor there.

To investigate on aging in the bonding zone we stored this substrate at laboratory atmosphere for a week. We re-suspended the substrate and measured the quality factor. The $Q$ decreased to $2.2 \times 10^{6}$. Then we replaced this fibre by a new one and measured the $Q$ again to be $2.6 \times 10^{6}$. After two months at laboratory atmosphere the $Q$ decreased to $6.6 \times 10^{5}$. We attribute this decrease of the $Q$ value to some aging of the bonding zone where the limiting losses occur. For instance the $\mathrm{H}_{2} \mathrm{O}$ produced by the hydrogen-burner can open micro-cracks in the pin material. To test this assumption we removed the pin and connected the fibre to the substrate using method 2 . The measured $Q$ increased to $1 \times 10^{6}$.

The internal mechanical quality factor for the nonmonolithic suspension was $2.5 \times 10^{5}$. This was the lowest $Q$ measured by us.

The normalized decay traces of the suspensions with the highest internal $Q$ for the different methods are shown in Fig. 2. Every trace is an average of several measured decays. The resonance frequency, the averaged decay time and the calculated internal $Q$ for these suspensions are presented in Table 1.

\section{Conclusion}

It was shown by Braginsky et al. that the mechanical $Q$ of the pendulum mode of monolithic fused silica suspensions can be very high. We have investigated monolithic suspensions for synthetic fused silica substrates with emphasis on very high mechanical quality factor of the internal modes of the substrates.

We considered three methods of producing these

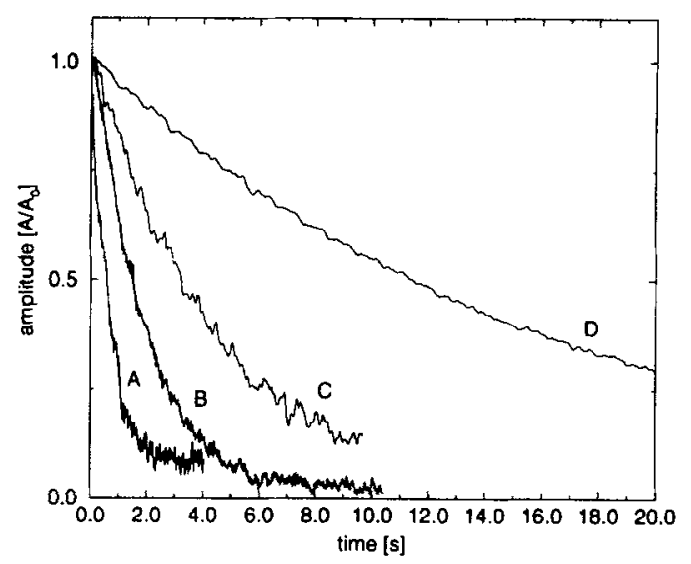

Fig. 2. Averaged decay traces (normalized) for suspensions produced with the bonding methods described in the text. See Table 1 for experimental data of the traces.

suspensions. With the first method we reached $Q$ values of $10^{5}$, by developing two other methods we reproducibly measured internal $Q$ 's of $10^{6}$. With proper fusing techniques, mechanical quality factors of $5 \times 10^{6}$ were achievable for the internal modes of the substrates in such a system.

We showed that the internal $Q$ in our suspensions was not limited by surface losses due to the treatment of the substrates with diamond tools. Losses due to bending within the bonding zone where thermal stress occurs are believed to be the limiting dissipation processes.

We have seen a significant lowering of the internal $Q$ if we stored the prepared substrates at laboratory atmosphere. We attribute this lowering to aging in the bonding zone. The aging processes and the possibilities of preventing it should be investigated.

Within the next future the use of our techniques to suspend large-scale test mass substrates will be investigated. The possibility of reaching very high mechanical quality factors in all mechanical modes support the idea of using monolithic test mass suspensions in laser-interferometric gravitational-wave detectors to lower the thermal noise contribution in the frequency band of interest. 


\section{Acknowledgement}

We wish to thank our colleagues in the Gravitational Wave Groups at the Universities of Glasgow and Hannover and at the Max-Planck-Institut für Quantenoptik for their interest in this work.

\section{References}

[1] K. Danzmann et al., GEO600 - Proposal for a $600 \mathrm{~m}$ LaserInterferometric Gravitational-Wave Antenna, Max-PlanckInstitut für Quantenoptik, report 190 (1994).

[2] A. Abramovici et al., Science 256 (1992) 325.
[3] C. Bradascia et al., Nucl. Instrum. Meth. Phys. Rev. A 289 (1990) 518

[4] P.R. Saulson, Phys. Rev. D 42 ( 1990) 2437

[5] V.B. Braginsky, V.P. Mitrofonov and O.A. Okhrimenk, Phys. Lett. A 175 (1993) 82.

[6] J.E. Logan, N.A. Robertson, J. Hough and P.J. Veitch, Phys. Lett. A 161 (1991) 101.

17] A. Gillespie, Thermal noise in the initial LIGOinterferometer, Ph.D. thesis, California Institute of Technology (1995).

[8] A. Gillespie and F. Raab, Phys. Lett. A 190 (1994) 213.

[9] J.E. Logan, N.A. Robertson and J. Hough, Phys. Lett. A 170 (1992) 352 .

[10] J. Hough, S. Rowan and S. Twyford, private communication. 